\title{
Mujeres dramaturgas en Buenos Aires: Mariana "Cumbi" Bustinza, su formación y producción en la escena porteña
}

Fecha de recepción: 24/02/2021. Fecha de aceptación: 20/03/2021.

En el teatro de Buenos Aires el rol de la mujer en la producción dramatúrgica fue muchas veces invisibilizado por las instituciones. A partir de diferentes investigaciones, en la actualidad podemos dar cuenta de su importancia en el desarrollo del teatro, considerando el aporte diverso de actrices, productoras, técnicas y escritoras. Este artículo se focaliza en Mariana "Cumbi" Bustinza, dramaturga en ejercicio actualmente. Para ello se reflexiona sobre su formación, dando cuenta de sus vínculos con la cumbia y la danza. De ese modo el trabajo intenta evidenciar algunas razones de su reconocimiento a nivel nacional.

PALABRAS CLAVE: DRAMATURGAS, FORMACIÓN, PRODUCCIÓN, TEATRO DE BUENOS AIRES, CUMBIA.

\section{Women Playwrights in Buenos Aires. Mariana "Cumbi" Bustinza, her Training and Production in the Buenos Aires Scene}

Women's role in drama production has many times been overlooked by Buenos Aires theatre institutions. Based on previous researches, nowadays it's possible to establish their place in drama development, considering the contribution of actresses, female producers, technicians and writers. This study is focused on Mariana "Cumbi" Bustinza, a female playwright who is currently working. We will reflect about her training, her bond with cumbia music and dancing. In this way, the study tries to make the reasons of her national popularity visible

KEYWORDS: PLAYWRIGHTS. PRODUCTION. TRAINING. THEATER OF BUENOS AIRES. CUMBIA.

\section{Introducción}

Este artículo se enmarca dentro de una de las líneas del proyecto FiloCyT "La formación en dramaturgia: construcción de un campo de saberes específico en la historia del teatro argentino", la cual forma parte de una investigación más amplia y aún en 
desarrollo sobre las relaciones entre la dramaturgia y la mujer en el ámbito local. En este sentido, la idea es examinar cuál fue el proceso formativo y las condiciones de producción que atravesaron diferentes mujeres, entre las cuales destacamos aquí la figura de Mariana "Cumbi" Bustinza.

Como es de notar, este trabajo se centra en una figura femenina que ha sido poco estudiada y cuya labor en la escena porteña se encuentra en pleno auge. Bustinza es una joven dramaturga que intenta, desde su poética, poner en escena la precariedad de los barrios marginales, en donde la cumbia se constituye en un elemento que los atraviesa.

En el teatro argentino el reconocimiento del trabajo de las mujeres ha sido escaso con relación al de los hombres, sobre todo en el área de la dramaturgia, y no porque no hayan estado presentes, sino porque gran parte de la historia las ha invisibilizado.

\section{Contextualización: las mujeres a lo largo de la historia del teatro argentino}

Antes de centrarnos en nuestra dramaturga elegida para este trabajo, es importante tener en cuenta el contexto en el que las mujeres dramaturgas de Argentina se han desarrollado. Susana Tarantuviez en Mujeres y teatro: el lugarde las mujeres en la historia del teatro argentino cuenta que en los primeros tiempos de nuestro teatro:
(...) la mujer ha estado presente (...) desde la época del virreinato. En efecto, si bien cuando se inaugura el teatro de la Ranchería, en 1783 únicamente actuaban hombres, quienes representan tanto los personajes masculinos como femeninos, pronto se incorporan las mujeres a las tablas. En este teatro de la Ranchería trabajan actrices profesionales, pero su oficio les acarrea un estigma social: son mal vistas por trabajar fuera de su hogar y en un oficio sobre el que pesaba la nota de infamia, que recién se abolirá en 1821. (Tarantuviez; 2013:169-170).

La presencia de las mujeres en el teatro en su rol de dramaturgas llegará tiempo después, cuando unas pocas mujeres pertenecientes a la alta sociedad de nuestro país, en su gran mayoría, serán las que se atrevan a escribir teatro, aunque algunas lo harán bajo seudónimos masculinos y otras directamente con el nombre de sus esposos.

Dentro de los pocos estudios que hay sobre la presencia de dramaturgas en el teatro nacional, se puede rescatar el realizado por la dramaturga, directora, actriz, docente, investigadora y artista plástica María Rosa Pfeiffer en su artículo Género chico (2019), donde realiza una división de cinco etapas históricas de visibilidad de las mujeres dramaturgas. La primera de ellas se da a comienzos del siglo XX, en la cual se destacan, entre otras: Lola Pita de Martínez, Alcira Olive, Alcira Chávez de Vila Bravo, Alfonsina Storni y Salvadora Medina de Onrubia. Una segunda etapa se desarrolla entre los años 40 y 80 -es importante el rescate de autoras que son mencionadas en el Diccionario de autores teatrales argentinos de Perla Zayas de Lima (2006)-. La tercera etapa abarca los años 80 , donde el período de dictadura va a ser un factor importante, ya que desemboca en tres ejes relevantes considerados por otra investigadora, Magda Castellvi deMoor, tales como las metáforas políticas, la reflexión del proceso de redemocratización y la exploración de problemáticas sobre la mujer. La cuarta etapa inicia en el 2002 a partir de la convocatoria de la autora Adriana Tursi, que invita a mujeres que escriben teatro a gestar Dramaturgas I, ejemplar que surge como cuestionamiento a una publicación del Instituto Nacional del Teatro enfocada en dramaturgos argentinos, que no contemplaba la presencia de ninguna mujer. Para el proyecto de Tursi se suman Susana Torres Molina, Liliana Cappagli, Patricia Suárez, 
Beatriz Mosquera, entre otras. Por último, la quinta etapa es desde el año 2014, cuando se realiza el Ciclo Autoras Argentinas en el Teatro Nacional Cervantes, para el cual la misma Adriana Tursi junto con Patricia Suárez se proponen realizar, en un formato de teatro semimontado, la lectura de 20 obras dramáticas.

En la actualidad existe el Colectivo de Autoras que nace en 2018 a raíz de que se conociera que solo el $1 \%$ de mujeres pertenecían a la dramaturgia en el circuito oficial nacional (a diferencia de lo que sucede en el circuito independiente donde se puede ver mayor producción de autoras). El colectivo existe gracias a la labor realizada por Adriana Tursi, Lucía Laragione, Susana Torres Molina y Mariela Asensio, y su objetivo está orientado a lograr una mayor visibilización de las mujeres autoras, con el fin de requerir la igualdad de derechos y oportunidades en todas las áreas en las que se desempeñan profesionalmente tanto dramaturgas, como también guionistas, coreógrafas y compositoras, buscando además paridad de género en los consejos asesores de los teatros oficiales, en los jurados de los concursos y en los órganos directivos de los organismos estatales de fomento cultural. Cabe destacar que este colectivo se maneja en sus bases de manera horizontal y federal, lo que quiere decir que incluye a mujeres de todo el país.

Es importante para este colectivo, la presencia de mujeres en las bibliografías de las diferentes instituciones educativas, ya que los espacios de formación introducen una clara ideología excluyente. En este sentido, podría decirse que Griselda Gambaro es la dramaturga que más se estudia dentro de estos espacios, quien a su vez es un faro de luz para las nuevas autoras.

La dramaturga en la que nos vamos a enfocar aquí, Mariana "Cumbi" Bustinza, también forma parte de este colectivo desde los inicios del mismo, apoyando las diferentes actividades y propuestas que se realizan. Dentro del nuevo mar de mujeres dramaturgas, Mariana Bustinza decide escribir sobre temáticas relacionadas con la marginación social invisibilizadas dentro del campo teatral.

\section{Mariana "Cumbi" Bustinza: creando desde los márgenes}

Mariana "Cumbi" Bustinza es una actriz, bailarina, cantante, profesora, directora y dramaturga porteña, cuya poética va a estar atravesada por sus vivencias personales dentro de los barrios marginales de la capital y del conurbano bonaerense, como así también se interesará por la cumbia, de tal modo que incluso es su gusto por este género musical el que le va a otorgar su apodo.

A lo largo de su carrera Bustinza ha sido galardonada con varios premios: en 2016 ganó una Estrella de mar por Mejor Labor Cómica Femenina, como así también Mejor Espectáculo de Humor por Dillinger, una comedia de su compañía Improvisa2. Asimismo, Menea para mí se llevó una Estrella de mar en el 2019 como Mejor Obra Alternativa y, por último, en el 2020 fue nominada Lo que quieren las guachas a los premios Hugo como Mejor Musical Off.

Bustinza se formó en la Escuela Metropolitana de Arte Dramático (EMAD) egresando de la carrera de actuación. Dentro de esta formación se especializó y dedicó durante mucho tiempo a la improvisación, en donde dio sus primeros pasos como dramaturga entendida su dramaturgia de actriz como una construcción en vivo de escritura dramática . Fundó su propia compañía Improvisa2 en el 2003, en la que se desempeñó como actriz, dramaturga, docente y directora, haciendo funciones y brindando talleres de impro -técnica canadiense-, por toda la Argentina e incluso en el exterior. Con dicha compañía participó también en Teatro x la 
Identidad. Ahora bien, cabe aclarar que no nos vamos a detener en este trabajo de su labor como actriz, dado que lo que nos interesa es su rol como dramaturga y directora dramática.

En el 2008, Bustinza decidió enfocarse en la dramaturgia para lo cual realizó un curso de cuatro meses, brindado por Cecilia Propato. Volvió a retomar su formación en el 2011, con Guillermo Hermida, durante dos meses. Y, en el 2016, decidió realizar el Curso de Dramaturgia de la EMAD, del cual se egresó en el 2018. Allí se formó con docentes, tales como Marina Jurberg, Camila Mansilla, Mauricio Kartun e Ignacio Apolo.

Como hemos visto, Bustinza acarrea consigo una larga trayectoria en el ámbito de la actuación, además de haber explorado el mundo de la dramaturgia a través de los cursos antes mencionados. Aun así, esto no impidió que se le presentara una dificultad a medida que pretendía abordar una poética propia, ya que su escritura no era lo que sus docentes pretendían. Este conflicto la llevó a repetir el primer año del curso de dramaturgia, aunque esto no impidió que siguiera adelante y lograra egresar del mismo. El recorrido de Bustinza por esta institución, la ayudó a afianzarse y así tomar seguridad en el terreno de la escritura.

A su vez, también el ejercicio de estar en constante práctica de la escritura le sirvió para darle linealidad y coherencia a su idea inicial. Si bien todavía no llevó a escena ninguna de las obras teatrales escritas en el marco del curso de Dramaturgia en la EMAD, su idea es hacerlo en años venideros, ya que tiene textos teatrales sumamente potentes.

Mariana "Cumbi" Bustinza, además de ser actriz y dramaturga egresada de la EMAD, ha estudiado danza teatro en la UNA. Esta formación se ve reflejada en sus obras, sobre todo en Menea para mi y Lo que quieren las guachas, puesto que ambas incluyen canciones y coreografías diseñadas por ella.

En 2015, por fuera de improvisa2, decide poner en escena la primera parte de su trilogía, Menea para mí, presentada en la sala teatral porteña El Extranjero, ganando con ésta el premio Teatro XXI, otorgado por el GETEA (Grupo de Estudios de Teatro Argentino e Iberoamericano), de la Universidad de Buenos Aires, lo cual le dio una importante legitimidad a su obra.

Los teatros independientes son espacios donde la mayoría de las dramaturgas encuentran un lugar para presentar sus obras. Como lo indica un relevamiento hecho por La Colectiva de Autoras en el 2018, en el circuito independiente el porcentaje de mujeres dramaturgas aumentó del 36\% en el 2015 al 42,7\% en el 2018. Dentro del circuito independiente, además, El extranjero es un teatro legitimado y es, a su vez, un espacio que brinda lugar a muchas dramaturgas (como, por ejemplo, a Mariela Asensio). En este espacio, Bustinza, en distintos momentos, ha llevado a escena dos de sus obras, Menea para mi y Lo que quieren las guachas. Por su parte, Gorila tuvo su última presentación en el Sportivo Teatral.

Estas obras forman parte de la trilogía que Bustinza ha desarrollado durante este último tiempo: Menea para mí, el amor en los barrios bajos (2015), Gorila (2016) y Lo que quieren las guachas (2019). Por lo general, presentan una temática que se corresponde con la realidad social actual y ponen de manifiesto elementos que forman parte de la cultura popular como, por ejemplo, el Gauchito Gil y la cumbia villera.

En estas obras incorporó la temática social como reflejo de sus propias vivencias. Las temáticas que se manifiestan se vinculan con la realidad social de los barrios más 
precarizados de la ciudad y el conurbano bonaerense, la discriminación, el aborto clandestino, el alcoholismo, el abuso en las infancias, la polaridad entre los que tienen y los que no, el abuso de poder, la invasión de bichitos colorados en casas precarias, la falta de justicia, las adicciones, la violencia familiar, la violencia de género, y la lista de faltas, necesidades y carencias sigue.

Después de investigar sobre el tema y de procesar muchas de sus vivencias personales, transformó ese material en un texto dramático titulado Menea para mí, donde se cruzan su formación en danza y su amor por la cumbia. La obra nos presenta la vida de siete adolescentes en un barrio marginal, quienes al ritmo de la cumbia nos van contando su vida dentro del mismo. Aquí se ponen de manifiesto el amor que se vive en la adolescencia, la familia disfuncional y el abuso, un padre ausente que vende “(...) paquito pa' los villeros" (Bustinza, 2015: 4), tal como lo relata Maxi -protagonista de la historia-, un padre que abusa de sus hijas y que golpea a su madre. A su vez, se exhibe el abuso de poder por parte de la policía, el gatillo fácil y la fe ciega en los santos paganos y populares como el Gauchito Gil.

Gorila se desarrolla en un barrio del conurbano y lo que se pone de manifiesto es la discriminación dentro de la misma clase social y a partir de relaciones de poder, como veremos más adelante. La Marlen y el Polaco se acaban de mudar desde la villa a un departamento en el que tienen como vecinas a Juana y Jimena, madre e hija. Esta última es una adolescente que asiste a un colegio privado, lugar al que siente que no pertenece debido a que no tiene lo mismo que sus compañeros. Pero, a su vez, tampoco acepta a sus nuevos vecinos a los que considera como negros, monos y gorilas.

Aquí la autora muestra la falta de conciencia de clase, que es reforzada por la idea de que Jime tiene acceso a una educación privada y escribe poemas, algo que contrasta con sus nuevos vecinos, quienes no recibieron la misma educación que ella. Un primer momento en el que Jimena pone en evidencia esta diferencia es cuando les lee un verso que escribió, el cual, a su vez, es un versito para niños: "azabache se cansó de oírse llamar negrito/ tomo la tiza y se blanqueó la cara y las dos manitos/ salió a la calle el negrito muy contento con su cambio/ pues estaba muy seguro que todos lo creían blanco". A lo que el Polaco responde: "bien... qué bien... te reee piola, para los pibitos ¿no?" (Bustinza, 2016: 13), sin comprender realmente lo que este poema quería decir.

El momento más álgido de esta obra, en el que se resaltan las diferentes relaciones de poder, es cuando durante una discusión entre el Polaco y Jimena, ésta le dice:

(...) Hay que prenderlos fuego y rociarlos con ácido sulfúrico y torturarlos infinitamente antes de su muerte, electrocutarlos, violarlos, golpearlos, forrearlos, apuñalarlos, contagiarles SIDA, y todos los peores males sobre la faz de la tierra, para por fin, asesinarlos de la manera más tortuosa y dolorosa que exista y que sufran en exceso... villero villero villero (Bustinza, 2016: 26).

Es durante esta discusión, en la que Jimena una vez más da cuenta de su superioridad y su deseo de ascenso social, que se desencadena la furia del Polaco y este decide marcar su fuerza, poder y superioridad como varón, asesinándola. Es decir, la obra pone en escena el femicidio de la joven.

Por último, Lo que quieren las guachas marca la diferencia entre los que tienen dinero y los que no. Dicha división de mundos va a estar dada por paredones: por un lado, un colegio privado y, por otro, una casa en una villa. Del lado del colegio, hay tres adolescentes, Sol, Mica y Valentino y, del otro lado, tenemos a una madre travesti llamada Mariela, con sus dos hijos, Owen y Yanina. 
Micaela y Valentino son novios pero terminan la relación debido al constante abuso de este último. Owen, que vende medias de manera ambulante, conoce a Mica en ese paredón, se enamora de ella y la invita a salir. Esto es algo que despierta la violencia en su ex Valentino, por lo cual, a modo de venganza, decide conquistar a Yanina solo con el afán y el deseo de abusar y mofarse de ella. En cierto momento, ambas jóvenes quedan embarazadas. Lo que desencadena diferentes maneras de actuar en los protagonistas. Por un lado, tenemos a Owen que está presente, acompañando a Mica y se pone feliz de tener un hijo con ella; y, por otra parte, tenemos a Valentino que rechaza la paternidad y echa a Yanina cuando esta lo busca en el colegio.

Ahora bien, lo que hace a continuación la autora es dar cuenta de las diferencias que implica el aborto clandestino (recordemos que la obra es del 2019 y en ese momento el aborto no era legal en la Argentina). Por un lado, tenemos a Mica, a la cual su madre la lleva a un consultorio privado y aborta, sin el consentimiento de ésta, de manera segura y con todos los cuidados que este procedimiento implica. Por el otro lado, tenemos a Yanina que junto con su madre recurren a la persona que realiza los abortos dentro de la villa, lo que termina desencadenando la muerte de la joven por el sangrado excesivo.

Como podemos observar, las obras de la autora visibilizan, entonces, diferentes problemáticas que no encontramos habitualmente en el teatro, dando cuenta del contexto actual en el que vivimos, logrando de esta forma configurar una mirada crítica sobre esta realidad. Sin embargo, Bustinza logra interpelar utilizando, según sus propias palabras, "el lenguaje de la danza, el teatro y la cumbia, para escapar un poco de esta realidad, pero sabemos que esto es real, esto sucede" (Bustinza, 2019b).

\section{A modo de conclusión}

Para comprender el lugar de las mujeres en el teatro de Buenos Aires es central ver el contexto en el que las dramaturgas de Argentina se han desarrollado. Primero hemos visto cómo las mujeres formaron parte del teatro como actrices y cómo recién a comienzos del siglo XX se han incorporado como dramaturgas, avanzando hacia la visibilización en ese medio. En la actualidad, y a partir del avance del movimiento feminista, existen diferentes colectivos que agrupan a mujeres en el teatro según su rol. Como ya se ha dicho, cabe destacar que Mariana "Cumbi" Bustinza participa de la Colectiva de Autoras, generando redes con el fin de lograr una mayor visibilización de las mujeres autoras y requerir la igualdad de derechos y oportunidades en todas las áreas en las que se desempeñan profesionalmente. Ya no se trata solamente de reunirse para sacar adelante una publicación de textos producidos por mujeres, sino que se trata de una búsqueda de transformación social asumiendo el compromiso de visibilizar ciertas zonas de la sociedad que fueron durante mucho tiempo marginadas. Así podemos afirmar que cada vez es mayor la visibilización de las mujeres en el teatro y, particularmente, en la dramaturgia del teatro de Buenos Aires. El caso de Bustinza resulta de este modo un "caso testigo" de esta situación transformadora. En esa línea, resulta necesario destacar que este trabajo se proyecta como fragmento parcial de un panorama mucho más abarcador, ya que son muchas las mujeres que forman parte de la dramaturgia en nuestro país y que siguen pugnando para lograr una mayor visibilidad a partir de la creación de sus poéticas personales. 


\section{Q Bibliografía}

» Bustinza, M. “Cumbi” (2015). Menea para mí. El amor en un barrio bajo. Mimeo cedido por la autora.

» Bustinza, M. “Cumbi” (2016). Gorila. Mimeo cedido por la autora.

»Bustinza, M. “Cumbi” (2019a). Lo que quieren las guachas. Mimeo cedido por la autora.

» Bustinza, M. “Cumbi” (2019b). Entrevista por comunicación personal [26 de septiembre de 2019].

»Castellvi de Moor, M. (1995). "Espacios femeninos en la dramaturgia argentina”, en Pellettieri, O. (ed.). El teatro y los días: Estudios sobre teatro iberoamericano y argentino, Buenos Aires, Galerna, 175-185.

»Pinta, M. F. (2006). “Género y escritura dramática en el teatro argentino”. Bibliográfica al libro de Magda Castellvi deMoor. Dramaturgas argentinas, teatro política y género. Facultad de Filosofía y Letras, Universidad Nacional de Cuyo, Mendoza, Argentina, 2003. En Telón de Fondo, revista de teoría y crítica teatral. www.telondefondo.org

»Pfeiffer, M. R. (2019). “Género chico”. El hilo de la fábula, Vol. 19, pp. 181-185. En: https://bibliotecavirtual.unl.edu.ar/publicaciones/index.php/HilodelaFabula/ article/view/8644/12065

» Santoro, S. (2002). “Dramaturgas”. Las 12. Página 12. En: https://www.pagina12. com.ar/diario/suplementos/las12/13-253-2002-07-05.html

» $s / f(2019)$. "Crean la Colectiva de Autoras para la igualdad de género en el teatro" Conclusión. En: https://www.conclusion.com.ar/espectaculos/crean-la-colectiva-de-autoras-para-la-igualdad-de-genero-en-el-teatro/o3/2019.

»Tarantuviez, S. (2013). “Mujer y teatro: el lugar de las mujeres en la historia del teatro argentino”. Revista Melibea. Vol.7. pp. 167-182. 